\title{
A novel cysteine-rich neurotrophic factor in Aplysia facilitates growth, MAPK activation, and long-term synaptic facilitation
}

\author{
Lu Pu, ${ }^{1,2}$ Ashley M. Kopec, ${ }^{1,2,3}$ Heather D. Boyle, ${ }^{1,2}$ and Thomas J. Carew ${ }^{1,2,3,4}$ \\ ${ }^{1}$ Department of Neurobiology and Behavior, University of California-Irvine, Irvine, California 92697, USA; ${ }^{2}$ Center for the \\ Neurobiology of Learning and Memory, University of California-Irvine, Irvine, California 92697, USA; ${ }^{3}$ Center for Neural \\ Science, New York University, New York, New York 10003, USA
}

\begin{abstract}
Neurotrophins are critically involved in developmental processes such as neuronal cell survival, growth, and differentiation, as well as in adult synaptic plasticity contributing to learning and memory. Our previous studies examining neurotrophins and memory formation in Aplysia showed that a TrkB ligand is required for MAPK activation, long-term synaptic facilitation (LTF), and long-term memory (LTM) for sensitization. These studies indicate that neurotrophin-like molecules in Aplysia can act as key elements in a functionally conserved TrkB signaling pathway. Here we report that we have cloned and characterized a novel neurotrophic factor, Aplysia cysteine-rich neurotrophic factor (apCRNF), which shares classical structural and functional characteristics with mammalian neurotrophins. We show that apCRNF (1) is highly enriched in the CNS, (2) enhances neurite elongation and branching, (3) interacts with mammalian TrkB and p75 ${ }^{\mathrm{NTR}}$, (4) is released from Aplysia CNS in an activity-dependent fashion, (5) facilitates MAPK activation in a tyrosine kinase dependent manner in response to sensitizing stimuli, and (6) facilitates the induction of LTF. These results show that apCRNF is a native neurotrophic factor in Aplysia that can engage the molecular and synaptic mechanisms underlying memory formation.
\end{abstract}

[Supplemental material is available for this article.]

The processes of development and memory formation both involve growth and reorganization of synaptic connections. Thus it is often suggested that they share common molecular mechanisms (Carew et al. 1998; Bailey and Kandel 2008). Supporting this view, extensive evidence indicates that the neurotrophin family of growth factors regulates both neuronal cell survival and differentiation in development, as well as synaptic plasticity and learning and memory in adult animals (Poo 2001; VicarioAbejon et al. 2002). In most species, neurotrophin signaling cascades are typically mediated by receptor tyrosine kinases (e.g., Trk A/B/C in mammals) and pan-neurotrophin receptor $\left(\mathrm{p} 75^{\mathrm{NTR}}\right.$ ) (Bibel et al. 1999; Chao 2003).

Previous studies have demonstrated a critical role for neurotrophin signaling in Aplysia californica (Purcell et al. 2003; Ormond et al. 2004; Sharma et al. 2006; Kassabov et al. 2013), a powerful model system for mechanistic studies of learning and memory formation (Bailey et al. 1996). For example, the induction of both long-term memory (LTM) and one of its cellular correlates, long-term synaptic facilitation (LTF), is facilitated by the activation of a tyrosine kinase-MAPK signaling pathway (Purcell et al. 2003). MAPK can be viewed as a molecular node (Reissner et al. 2006) that is a broker for growth factor signaling, including TrkB signaling, and is critical in learning and memory in Aplysia and other animals (Atkins et al. 1998; Reissner et al. 2006). Moreover, the TrkB ligand BDNF facilitates serotonin (5HT)-induced LTF in a MAPK-dependent manner, suggesting the existence of an endogenous TrkB-like receptor in Aplysia (Purcell et al. 2003; Sharma et al. 2006). Supporting this idea, inhibition of tyrosine kinase ac-

\footnotetext{
${ }^{4}$ Corresponding author E-mail tcarew@nyu.edu

Article is online at http://www.learnmem.org/cgi/doi/10.1101/lm.033662.113.
}

tivity blocks induction of LTF (Purcell et al. 2003). Furthermore, CNS application of a TrkB-Fc chimera, which sequesters endogenous BDNF-like ligands, blocks the activation of MAPK, the induction of LTF, and the formation of LTM in response to training with either 5HT or tail shock (TS) (Sharma et al. 2006). In Aplysia, two Trk receptors with conserved tyrosine kinase domains, ApTrkl and ApTrk, and an Aplysia neurotrophin ortholog, ApNT, have recently been cloned and shown to contribute to LTF (Ormond et al. 2004; Kassabov et al. 2013). These results demonstrate the existence of endogenous neurotrophic factors and their receptors in invertebrates which contribute to synaptic plasticity and memory formation.

The analysis of neurotrophic factors provides an excellent platform to explore the hypothesis that development and learning share common molecular mechanisms. To support this hypothesis in Aplysia, two basic conditions must be met. First, the neurotrophic factors in Aplysia should be released in the CNS and behave like bona fide growth factors. For example, they should promote neuronal growth (Poo 2001). Second, these neurotrophic factors should engage the same molecular and cellular mechanisms that are known to subserve memory formation in Aplysia. In the present study we report the identification of apCRNF, a novel neurotrophic factor in Aplysia, which is expressed in the CNS of Aplysia, and enhances sensory neuron (SN) neurite elongation and branching. Moreover, we demonstrate that apCRNF interacts with mammalian neurotrophin receptors and is released by a training

(C) 2014 Pu et al. This article is distributed exclusively by Cold Spring Harbor Laboratory Press for the first 12 months after the full-issue publication date (see http://learnmem.cshlp.org/site/misc/terms.xhtml). After 12 months, it is available under a Creative Commons License (Attribution-NonCommercial 4.0 International), as described at http://creativecommons.org/licenses/bync $/ 4.0 /$. 
analog that induces activity-dependent memory for sensitization (Shobe et al. 2009). Additionally, apCRNF induces MAPK activation in conjunction with a single tail nerve shock (TNS, a sensitizing stimulus that releases 5HT in the CNS; Marinesco and Carew 2002), an effect which requires tyrosine kinase activity, and facilitates the induction of LTF in conjunction with a single pulse of 5HT. Taken together, these data support the hypothesis that apCRNF is a novel endogenous neurotrophic factor in Aplysia that contributes to the induction of synaptic plasticity underlying memory formation.

\section{Results}

\section{Identification and characterization of apCRNF}

Fainzilber et al. (1996) originally identified a novel molluscan neurotrophic factor, Lymnaea CRNF (lyCRNF) by its interaction with p75 ${ }^{\mathrm{NTR}}$. This neurotrophic factor is released into Lymnaea hemolymph and CNS conditioned medium, induces neurite outgrowth, and up-regulates voltage activated calcium currents in Lymnaea motor neurons (MNs) (Fainzilber et al. 1996). To determine if a similar neurotrophic factor is endogenous to Aplysia, we used the lyCRNF nucleotide sequence as a template for our search and cloned a novel CRNF gene from an Aplysia CNS cDNA library (GenBank accession no. EB259651), which shares 41.0\% identity with $l y C R N F$. apCRNF protein consists of 123 amino acid residues, with a theoretical molecular weight of $18 \mathrm{kDa}$. The protein sequence alignment between full length apCRNF and lyCRNF exhibits $31.5 \%$ similarity and $22.3 \%$ identity, including 11 highly conserved cysteine residues (Supplemental Fig. 1A). Using the Clustal Omega multisequence alignment server, apCRNF was aligned against a panel of mammalian neurotrophins and invertebrate neurotrophic factors, which shows that apCRNF contains conserved cysteines essential for cystine-knot structure (Fig. 1A), a structural hallmark of neurotrophins (Wiesmann and de Vos 2001). Collectively, the sequence similarity of apCRNF to known invertebrate neurotrophic factors and mammalian neurotrophins and its potential containment of a cystine-knot structure raise the possibility that apCRNF may represent a bona fide neurotrophic factor in Aplysia.

\section{apCRNF is expressed in adult Aplysia CNS}

Neurotrophins are critically important for nervous system development and neuronal growth and survival in mammals (Poo 2001). If apCRNF is acting as a bona fide neurotrophic factor, it should be expressed in the CNS of Aplysia. To further characterize apCRNF, recombinant GST- and His-apCRNF was expressed and purified (Supplemental Fig. 1B). We then raised a custom anti-apCRNF antibody, which specifically recognized GST- or His-apCRNF as major bands of $\sim 43 \mathrm{kDa}$ or $\sim 20 \mathrm{kDa}$, respectively (Supplemental Fig. 1C); both are consistent with the molecular weights of the tagged recombinant proteins. Consistent with the predicted molecular weight of endogenous apCRNF, this antibody recognized a major band of $\sim 18 \mathrm{kDa}$ in Aplysia tissue extracts, indicating apCRNF is richly expressed in pleural-pedal ganglia and heart, and weakly expressed in body wall and buccal mass (Fig. 1B). To determine the expression of apCRNF in isolated SNs and MNs, we performed immunocytochemistry on cultured Aplysia neurons. Our results revealed that apCRNF is localized in the somata and neurites of both SNs and MNs (Fig. 1C). RT-PCR assay showed that apCRNF is expressed in both pleural and pedal ganglia (Fig. 1D). The pleural ganglia contain the somata and processes of the SNs, and the pedal ganglia contain the tail motor neuron (MN) somata and the SN synapses onto tail MNs (Sherff and Carew 2004). Moreover, fluorescence in situ hybridization

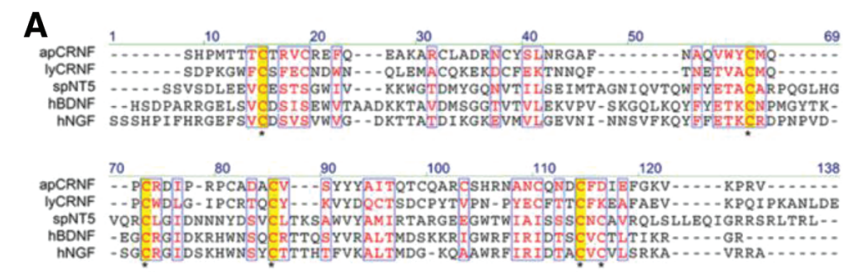

B
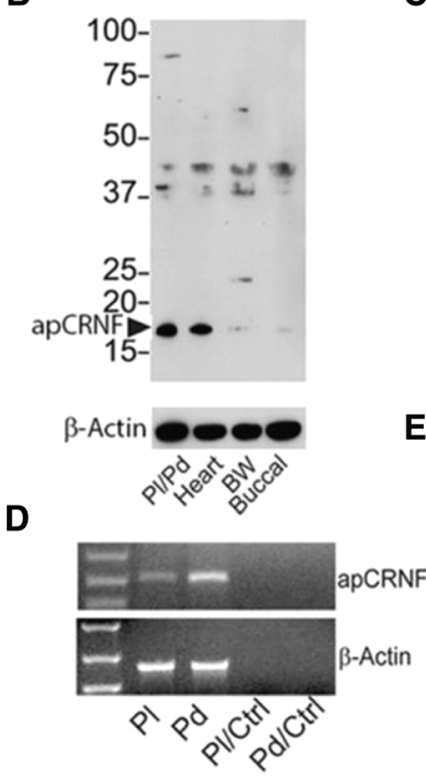

C

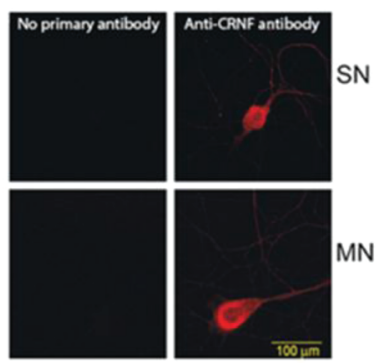

E
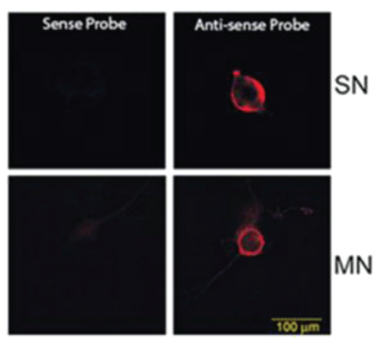

Figure 1. Cloning and characterization of Aplysia CRNF. (A) Structural alignment of cystine-knot domains of Aplysia CRNF (apCRNF), Lymnaea CRNF (lyCRNF), Stronglyocentrotus purpuratus (sea urchin) NT5 (spNT5), human BDNF (hBDNF), and human NGF (hNGF). The cysteines critical for the cystine-knot are highlighted in orange and labeled with a single asterisk $(*)$, and the conserved substitutions within the hydrophobic core are highlighted in boxes and shown in red. (B) Tissue distribution analysis reveals that apCRNF protein is expressed in CNS. (Top) apCRNF distribution determined by Western blot with anti-apCRNF antibody in the pleuralpedal ganglia (PI/Pd), heart, body wall (BW), and buccal mass. (Bottom) $\beta$-actin is used as a loading control. (C) Immunocytochemical analysis reveals that apCRNF protein is expressed in SNs and MNs. (Top panels) SNs; (bottom panels) MNs. Controls (without primary antibody) are shown on the left. (D) RT-PCR shows apCRNF mRNA levels in pleural (PI) and pedal $(\mathrm{Pd})$ ganglia. Control experiments in the absence of reverse transcriptase show no signal in either pleural ( $\mathrm{Pl} / \mathrm{Ctrl})$ or pedal ganglia $(\mathrm{Pd} /$ Ctrl). Actin is used as an internal control. (E) Fluorescent in situ hybridization shows apCRNF transcripts are expressed in SNs and MNs. (Top panels) SNs; (bottom panels) MNs. Controls with sense RNA probes are shown on the left.

(FISH) showed that apCRNF transcripts are expressed in both SN and MN cell bodies (Fig. 1E). These results are consistent with the pre- and postsynaptic localization of neurotrophins at mammalian synapses that exhibit activity-dependent plasticity (Poo 2001). Interestingly, ApNT, a recently characterized neurotrophin ortholog endogenous to Aplysia, is primarily localized to the sensory neurons (Kassabov et al. 2013), suggesting that ApNT and apCRNF may serve overlapping but distinct functional roles in the Aplysia CNS (see Discussion).

\section{apCRNF enhances SN neurite growth and branching}

In vertebrates, neurotrophins are critical for neurite growth, including neurite elongation, axonal arborization, dendritic 
branching, and spine formation (Levi-Montalcini and Angeletti 1968; Cohen-Cory et al. 2010). To examine the effect of apCRNF on neurite growth, we treated isolated Aplysia SNs in culture with GST-apCRNF $(250 \mathrm{ng} / \mathrm{mL})$ or vehicle $(0.1 \%$ BSA in PBS) for $3 \mathrm{~d}$. Images of the SNs were then taken and subjected to Sholl analysis (see Materials and Methods). The number of intersections between neurites and each ring (Fig. 2A,B) was independently counted using a blind procedure. The numbers of neurite crossings after apCRNF treatment $(n=12)$ was consistently greater than those of control ( $n=7$ ) (Fig. 2C). To quantify the aggregate data, we determined the areas under curves in Figure 2C (Gensel et al. 2010), and found that the area for apCRNF treatment was significantly larger than the area for the control treatment (two-tailed $t$-test, $\left.t_{(17)}=2.214, P<0.05\right)$ (Fig. 2D). These data indicate that apCRNF enhances neurite growth and branching of SNs and suggest the existence of an endogenous receptor for apCRNF.

\section{apCRNF interacts with rat TrkB and $\mathrm{p} 75^{\mathrm{NTR}}$}

Given that apCRNF shares sequence similarity with neurotrophins, we next asked if apCRNF can bind neurotrophin receptors, which may mediate the facilitatory effects on neurite outgrowth seen in Figure 2. As part of a Trk-p75 ${ }^{\mathrm{NTR}}$ receptor complex, Trk receptor tyrosine kinases can be activated and phosphorylated by the binding of neurotrophins, which leads to the transduction of a variety of downstream signaling events, including MAPK phosphorylation (Chao 2003). In addition, the presence of p75 ${ }^{\mathrm{NTR}}$ can enhance the neurotrophin-Trk affinity (Bibel et al. 1999). In the present study, we explored the possible interaction of apCRNF with mammalian neurotrophin receptors using a GST pull-down assay with recombinant GST-apCRNF protein (Supplemental Fig. 1B). Specifically, we asked if GST-apCRNF interacts with rat TrkB and $p 75^{\mathrm{NTR}}$, since (1) TrkB signaling is critical for learning and memory in Aplysia (Purcell et al. 2003; Sharma et al. 2006) and (2) all neurotrophins bind to p75 ${ }^{\text {NTR }}$ (Chao 2003). As shown in Figure 3, GST-apCRNF, but not GST alone, binds to both rat TrkB (Fig. 3A) and p75 ${ }^{\mathrm{NTR}}$ (Fig. 3B). These data implicate activation of neurotrophin receptor orthologs in Aplysia as a possible mechanism for mediating apCRNF's neurotrophic effects.

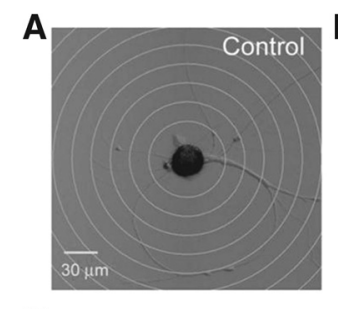

C

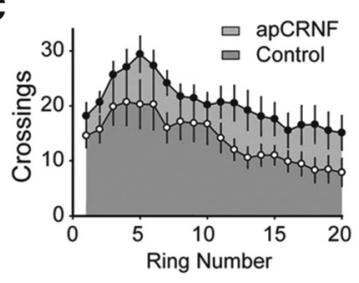

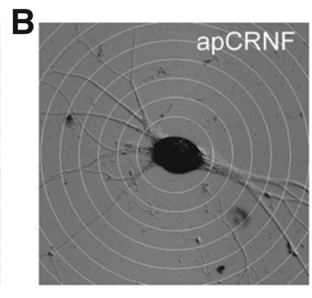

D

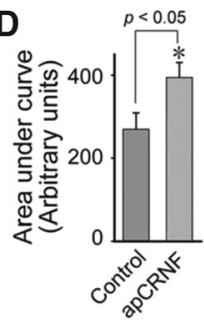

Figure 2. apCRNF increases neurite branching of Aplysia sensory neurons. Representative images of SNs after $3 \mathrm{~d}$ of vehicle $(0.1 \% \mathrm{BSA}$ in PBS $(A)$ or apCRNF $(250 \mathrm{ng} / \mathrm{mL})$ treatment $(B)$. A set of 20 rings of increasing radii was generated and superimposed on the bright field images. (C) Sholl analysis indicates that apCRNF treatment increases the number of SN neurite crossings compared to the control treatment. $(D)$ The area beneath the curve in $C$ was significantly larger for apCRNF treatment $(n=12)$ compared to control $(n=7)$.
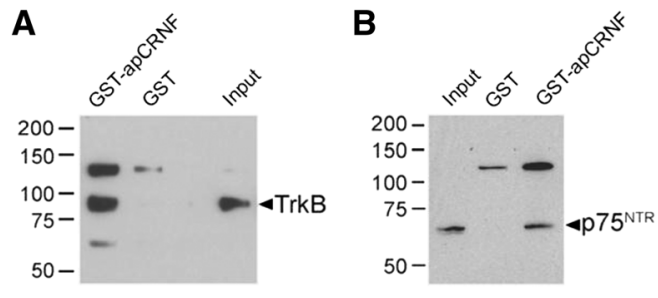

Figure 3. apCRNF interacts with mammalian TrkB and $p 75^{\mathrm{NTR}}$. Rat brain extract was incubated with recombinant GST or GST-apCRNF, and then TrkB $(A)$ or $p 75^{\text {NTR }}(B)$ was detected by Western blotting.

\section{apCRNF is released in the CNS in an activity-dependent fashion}

Because many neurotrophic factors play dual roles in development and adult plasticity, we next asked if apCRNF is engaged during the induction of plasticity in Aplysia. For a neurotrophic factor to contribute to learning and memory formation, it must (1) be released in response to plasticity-inducing stimuli, and (2) engage molecular and cellular substrates required for learning and memory formation. In previous studies, we found that the combination of a pulse of $\mathrm{KCl}$ (which depolarizes Aplysia SN membranes $\sim 20 \mathrm{mV}$ and induces calcium influx) and a pulse of 5HT can be used as a molecular analog of associative learning to induce MAPK activation and activity-dependent intermediateterm memory (Ye et al. 2008; Shobe et al. 2009). Using an enzymelinked immunosorbent assay (ELISA) for apCRNF, we observed a significant increase of apCRNF protein level in the conditioned medium of Aplysia pleural-pedal ganglia that received a pulse of $\mathrm{KCl}$ and $5 \mathrm{HT}(158.6 \pm 26.1 \%, n=10)$, compared to the control ganglia receiving ASW treatment $(100.0 \pm 16.3 \%$; one-tailed $t$-test, $t_{(18)}=1.903, P<0.04$ ) (Fig. 4). The release of apCRNF triggered by the combination of $\mathrm{KCl}$ and $5 \mathrm{HT}$ is consistent with related observations showing regulated secretion of NGF, BDNF, and NT3 (Poo 2001).

\section{apCRNF facilitates MAPK activation}

A single 5HT exposure or a single tail shock (TS) induces only shortterm plasticity, but not LTF/LTM in Aplysia (Sutton et al. 2002). In contrast, multiple TSs induce LTM in a MAPK dependent manner (Sharma et al. 2003). In addition, Purcell et al. (2003) showed that subthreshold training with 5HT or TS, when paired with up-regulation of tyrosine kinase signaling by bpV (a tyrosine kinase phosphatase inhibitor), is able to induce MAPK activation and LTM. If apCRNF is released by sensitizing stimuli in Aplysia and engages a receptor tyrosine kinase cascade (potentially mediated by a TrkB-like receptor), one would predict that sensitizing stimuli such as tail nerve shocks (TNSs) would give rise to activation of signaling molecules canonically downstream of tyrosine kinase activity, including MAPK activation (Huang and Reichardt 2003). To test this prediction, we examined MAPK activation induced by (1) apCRNF alone, (2) a single TNS, and (3) a combined treatment of apCRNF and a single TNS (CRNF $+1 \times$ TNS). Phospho-MAPK levels were normalized to total MAPK within the same sample, and then the relative amount of phospho-MAPK in the experimental group was compared to the within-animal control group. Neither apCRNF alone $(500 \mathrm{ng} /$ $\mathrm{mL})$ nor a single TNS alone (100.1 $\pm 16.3 \%, n=6 ; 118.5 \pm$ $13.2 \%, n=7$; NS) induced MAPK activation $1 \mathrm{~h}$ after the shock above within-animal control levels (Fig. 5A), a time point at which there should be no MAPK activation by a single sensitizing trial alone (Philips et al. 2007, 2013). In contrast, the combined treatment of apCRNF $(500 \mathrm{ng} / \mathrm{mL})$ and a single TNS induced significant 


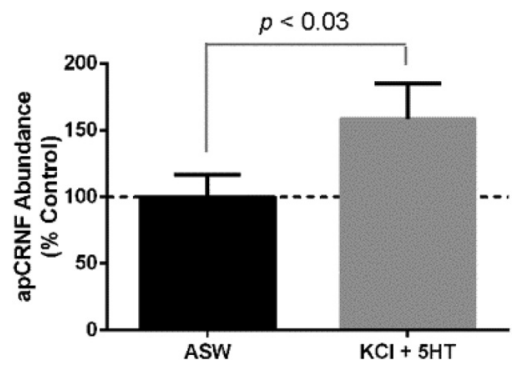

Figure 4. apCRNF is released by activity-inducing stimuli in the CNS. ELISA analysis shows that apCRNF protein level in conditioned medium of Aplysia pleural-pedal ganglia which received $\mathrm{KCl}+5 \mathrm{HT}$ treatment is significantly higher than that in control $(n=10)$.

MAPK activation above within-animal control levels (181.0 \pm $10.1 \%, n=12, P<0.01$ ) (see Materials and Methods). A one-way ANOVA revealed a significant difference among the three groups $\left(F_{(2,22)}=12.38, P<0.001\right)$. Subsequent planned comparisons indicated a significant difference between the apCRNF $+1 \times$ TNS group and the apCRNF alone (Fischer's LSD, $t_{(22)}=4.451, P<$ 0.001 ), and a single TNS alone (Fischer's LSD, $t_{(22)}=3.645, P<$ $0.01)$, respectively. Finally, we found that the facilitation of MAPK activation by apCRNF $+1 \times$ TNS requires tyrosine kinase activity: apCRNF $+1 \times$ TNS did not significantly increase MAPK activation above within-animal control levels (see Materials and Methods) in the presence of genistein $(100 \mu \mathrm{M})$, a general tyrosine kinase inhibitor (Wilcoxin matched-pairs signed rank test, $n=8, W=0, \mathrm{NS})$, but MAPK was significantly increased above within-animal control levels in the presence of vehicle $(0.33 \%$ DMSO $/ 0.43 \% \mathrm{EtOH}, n=6$; Wilcoxin matched-pairs test, $W=21$, $P<0.04$ ) (Fig. 5B). Collectively, these data show that apCRNF facilitates the induction of a tyrosine kinase-MAPK cascade in response to a subthreshold sensitizing stimulus.

\section{apCRNF facilitates the induction of LTF}

To explore the effect of apCRNF on synaptic plasticity, we examined LTF induction at cultured SN-MN synapses. EPSPs at $\mathrm{SN}-$ MN synapses were measured before and 18-24 h after apCRNF treatment. Neither apCRNF alone (median \pm IQR, $125 \pm 118, n=$ 5; Wilcoxin matched-pairs test, $W=9, P=0.31$ ) nor a single pulse of $5 \mathrm{HT}$ (median $\pm \mathrm{IQR}, 86 \pm 62, n=12$; Wilcoxin matched-pairs test, $W=-10, P=0.73$ ) induced significant facilitation from pretreatment baseline measurements $18-24 \mathrm{~h}$ after treatment (Fig. 6). However, following apCRNF-5HT pairing, significant LTF was induced relative to pretreatment baseline levels (median \pm IQR, $158 \pm 149, n=7$; Wilcoxin matched-pairs test, $W=28, P<0.02)$. Furthermore, Kruskal-Wallis ANOVA revealed that there was an overall significant difference among the groups $\left(\chi^{2}(2)=7.258, P<0.03\right.$ ) (Fig. 6B). Subsequent planned comparisons identified a significant difference between 5HT alone and apCRNF-5HT paired groups (Dunn's multiple comparisons test, Mean rank diff. $=-8.881, P<0.05)$. Intriguingly, there was not a significant difference between the apCRNF alone and apCRNF5HT groups (Dunn's multiple comparisons test, Mean rank diff. $=-3.714$ ), although only the apCRNF-5HTgroup (and not the apCRNF alone group) showed significant facilitation from baseline. Additionally, there appeared to be a trend toward synaptic facilitation with apCRNF alone treatment $(P=0.31)$, an observation reminiscent of a finding by Kassabov et al. (2013) who reported that ApNT alone can cause synaptic facilitation in Aplysia SN-MN coculture. In conclusion, consistent with our previous findings examining the facilitating effects of mam- malian BDNF (Purcell et al. 2003; Sharma et al. 2006), we here show that apCRNF also facilitates the induction of LTF at SNMN synapses.

\section{Discussion}

\section{Endogenous Aplysia growth factors}

In the present study, we report the cloning and characterization of a novel Aplysia neurotrophic factor, apCRNF. In addition to the functional similarities between apCRNF and known neurotrophins, Clustal Omega multi-sequence alignment shows that apCRNF possesses cysteines essential for a cystine-knot (Fig. 1A). A cystine-knot usually consists of six highly conserved cysteines forming three intramolecular disulfide bonds; it is characteristically found in neurotrophins such as NGF, BDNF, NT-3, and NT-4, and is critical for stabilizing their molecular conformation (Wiesmann and de Vos 2001). In addition, the tertiary structures of apCRNF and canonical neurotrophins also share considerable similarity. During evolution, orthologous proteins may have diverged significantly in primary sequences, yet their three-dimensional structures may remain conserved. FUGUE, a structure-based search algorithm, was developed to identify the structural similarity in three dimensions (Shi et al. 2001). Recently, novel Drosophila neurotrophins with structural conservation but sequence divergence from mammalian neurotrophins were identified using a FUGUE-based search (Zhu et al. 2008). We used FUGUE to compare apCRNF with mammalian neurotrophins with known crystal structures, including BDNF and NGF. FUGUE predicts that apCRNF belongs to the mammalian neurotrophin family with a confidence level that is $>99 \%$ (ZSCORE $=15.54)$. Although we do not have a definite structure for apCRNF, the FUGUE prediction supports the structural similarity between apCRNF and mammalian neurotrophins. Hence, despite the divergence of the apCRNF primary sequence, its structural and functional similarities to vertebrate neurotrophins, as well as its ability to interact with the TrkB and $\mathrm{p} 75^{\mathrm{NTR}}$ receptors, strongly indicate that apCRNF is a bona fide Aplysia neurotrophin.
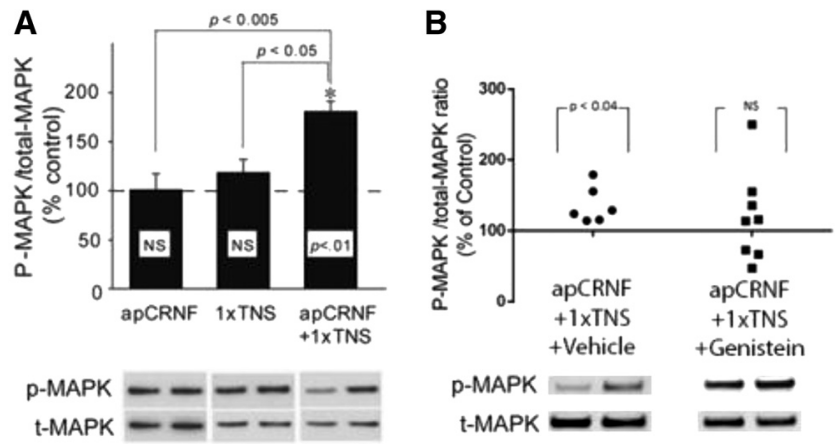

Figure 5. apCRNF facilitates the induction of a tyrosine kinase-MAPK cascade in SN clusters. (A) Summary data of MAPK activation induced by apCRNF $(500 \mathrm{ng} / \mathrm{mL}, n=6)$, a single TNS $(n=7)$, and apCRNF in combination with a single TNS $(n=12)$. Representative Western blots are presented below the summary data: phospho-MAPK (top) is normalized to total-MAPK (bottom), and then experimental phospho-total MAPK ratios (left) are compared to control phospho-total MAPK ratios (right). (B) Tyrosine kinase activity is required for MAPK activation induced by apCRNF $+1 \times$ TNS. Each circle (apCRNF $[500 \mathrm{ng} / \mathrm{mL}]+$ $1 \times$ TNS + Vehicle [0.33\% DMSO/0.43\% EtOH]) or square (apCRNF + $1 \times$ TNS + Genistein $[100 \mu \mathrm{M}])$ represents an individual experiment. There was significant MAPK activation in the vehicle group $(P<0.04)$, but not in the genistein group $(P>0.99)$. Representative Western blots are presented below the summary data as in $A$. 


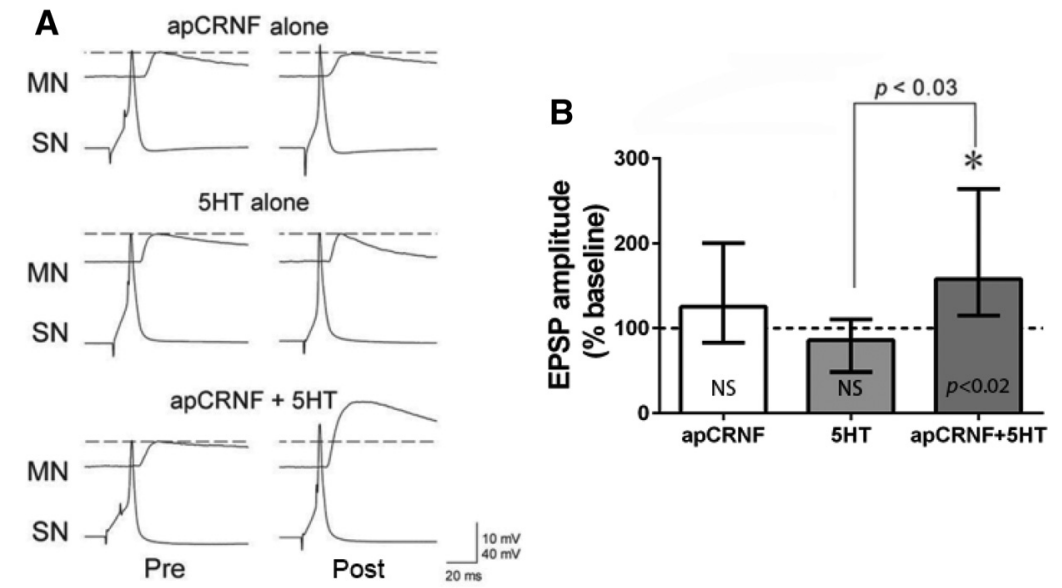

Figure 6. apCRNF facilitates the induction of LTF at SN-MN synapses. $(A)$ Representative traces from independent experiments before (Pre) and 18-24 h after (Post) apCRNF alone $(n=5)$, a 5-min pulse of $5 \mathrm{HT}$ alone $(n=12)$, and a 5 -min pulse of $5 \mathrm{HT}$ in combination with apCRNF (apCRNF $+5 \mathrm{HT}, n=7)$. $(B)$ Summary data indicating the normalized EPSP amplitude at the long-term test point (18-24 h after treatment). apCRNF or 5HT alone do not induce LTF, but in conjunction they induce significant LTF. Data are expressed as median EPSP \pm IQR.

In addition to our previous findings that BDNF-like molecules are critical in learning and memory in Aplysia (Purcell et al. 2003; Sharma et al. 2006), two additional Aplysia growth factors have been identified. In a recent seminal study, Kassabov et al. (2013) identified ApNT, an invertebrate neurotrophin ortholog belonging to the canonical vertebrate neurotrophin family. ApNT is expressed as both a pro- and mature neurotrophin based on different splice variants. Both forms of ApNT are secreted and participate in serotonin-induced LTF in Aplysia, although interfering with ApNT signaling alone does not appear to be sufficient to completely disrupt LTF (Kassabov et al. 2013). Interestingly, a clustal alignment indicates apCRNF and ApNT both contain conserved cysteines similar to mammalian neurotrophins (Supplemental Fig. 2). However, apCRNF and ApNT have distinct sequences, suggesting these neurotrophic factors could serve different functional roles in Aplysia. In earlier work, $\mathrm{Hu}$ et al. (2004) found that another growth factor-like molecule, sensorin, is located in SNs and is secreted in response to repeated pulses of 5HT. Similar to neurotrophins, the released sensorin is required for synapse formation and LTF, indicating its critical role in synaptic plasticity in Aplysia. However, with only two cysteines, sensorin cannot form the cystine-knot typical of neurotrophin family members. Consistent with this view, a FUGUE analysis predicts that the structure of sensorin is not similar to that shared between apCRNF and mammalian neurotrophins.

Other growth factor families have also been found to be important for plasticity in Aplysia. Zhang et al. (1997) discovered that transforming growth factor $\beta-1$ (TGF $\beta-1)$, a member of the TGF $\beta$ superfamily of growth factors, is required for LTF in Aplysia. Interestingly, TGF $\beta-1$ can rapidly activate MAPK (Chin et al. 2002), indicating that different growth factor families may contribute to synaptic plasticity through converging molecular mechanisms. Collectively, these results support the conclusion that there are multiple endogenous growth factor families in Aplysia that can participate in learning-related synaptic plasticity. Many growth factors employ distinct, but overlapping, signaling cascades to mediate similar functional outcomes (Kopec and Carew 2013). Thus it will now be particularly interesting to utilize Aplysia's well-characterized CNS to determine both the shared and perhaps unique contributions of different growth factor families to learning and memory formation.

\section{Endogenous Aplysia neurotrophin receptors}

The identification of different invertebrate neurotrophic factors naturally gives rise to a search for endogenous invertebrate neurotrophin receptors. In mammalian systems, there are three neurotrophin Trk receptors: NGF binds to TrkA, BDNF and NT4/5 to TrkB, and NT3 to TrkC (Chao 2003). Similarly, multiple neurotrophins and receptor tyrosine kinases may be involved in LTF and LTM in Aplysia and other invertebrate systems.

Although to date no Trk receptors have been reported in Drosophila or C. elegans, several Trk or Trk-like receptors have been identified in mollusks (Beck et al. 2004; Ormond et al. 2004; Kassabov et al. 2013). LTrk, a prototypical Trk receptor in Lymnaea, is required in neuronal survival and outgrowth (Beck et al. 2004). In Aplysia, in addition to the two aforementioned neurotrophins, ApNT (Kassabov et al. 2013) and apCRNF (described here), two Trk receptors, ApTrkl and ApTrk, have been identified (Ormond et al. 2004; Kassabov et al. 2013). ApTrkl is localized in Aplysia sensory neurons and is required for 5HT-induced MAPK activation and LTF. It has a highly conserved intracellular tyrosine kinase domain and a novel extracellular domain not homologous to the sequences of any known Trk receptors (Ormond et al. 2004). The mechanism of 5HT activation of ApTrkl, which might require the transactivation via G-protein coupled receptors, remains unknown (Ormond et al. 2004). Moreover, another Trk receptor, ApTrk, is localized to both SNs and MNs (and is particularly enriched in MNs), is capable of activating MAPK, AKT, and PLC $\gamma$ in PC12nnr5 cells upon stimulation with ApNT, and plays an important role in LTF in Aplysia SN-MN coculture (Kassabov et al. 2013). Collectively, an intriguing possibility for future exploration is that, in response to activity-inducing stimuli, apCRNF is released and interacts with one of the Aplysia neurotrophin receptors to elicit the canonical Trk-MAPK signaling cascades.

In an important recent study, McIlroy et al. (2013) showed that Drosophila neurotrophins DNT1 and DNT2 bind to Toll-6 and Toll-7 of the Toll receptor superfamily, critically important for mediating innate immunity responses. These data raise the interesting possibility that invertebrate neurotrophins may promiscuously bind to several different types of receptors to induce their trophic effects. It now will be important to determine if apCRNF is, indeed, activating one of the identified Trk receptors in Aplysia, or if it is perhaps acting through novel receptors such as Toll receptors, or as yet unknown receptors, to mediate plasticity.

\section{Mechanisms of Aplysia neurotrophin signaling in learning and memory}

More than a century ago, Ramón y Cajal raised the intriguing possibility that development and learning may have shared mechanisms (Carew et al. 1998; Bailey and Kandel 2008). Both neuronal development and memory formation are highly complex. Nevertheless, several processes have been proposed as critical points of contact for possible shared mechanisms of development and memory formation: (1) structural modifications of the synapse mediated by both target-oriented neuronal growth and synapse formation, (2) activity-dependent synaptic refinement in 
neural pathways via coincident activity, and (3) regulation of synaptic efficacy, including LTP- and LTD-like mechanisms (Carew et al. 1998; McKay et al. 1999). Interestingly, neurotrophin signaling is involved in all of these processes and is likely to be highly conserved in both vertebrates and invertebrates (Carew et al. 1998; Bailey and Kandel 2008). Considering these several principles, in the present study we present evidence consistent with the view that apCRNF is a functional Aplysia neurotrophic factor and may be a secreted TrkB ligand essential to both synaptic refinement and memory formation in Aplysia. Thus, apCRNF may serve as a strategic focal point for studying the shared mechanisms of development and learning and memory in Aplysia.

\section{Materials and Methods}

\section{Animals}

Wild-caught A. californica (150-250 g) were obtained from Marinus Scientific (Long Beach, CA), Charles Hollahan (Santa Barbara, CA), and South Coast Bio-Marine, LLC (Los Angeles, CA) and were dissected as described in Reissner et al. (2010).

\section{Cloning and recombinant protein expression}

apCRNF cDNA was isolated from an Aplysia CNS cDNA library by PCR, using primers 5'-CACCATGGCGGCAGAGTCCCA-3' and 5'-TGTTTACGAGTACATGAAGGGC-3'. For expression in bacteria, apCRNF was cloned into pDEST-15 (GST-tagged) or pDEST-17 (His-tagged) vectors. All constructs were verified by sequence analysis. GST- or His-apCRNF was expressed in Rosetta2 (DE3) pLys cells and purified using glutathione-agarose or His-binding resins as described in Reissner et al. (2008). Glutathione or imidazole was then removed using either dialysis or gel filtration. The purity of GST-CRNF was examined using SDS-PAGE with Coomassie Blue staining.

\section{Structure-based alignment}

Multiple sequence alignment of mature neurotrophins (pre and/ or pro sequences were removed) was performed using Clustal Omega (Sievers et al. 2011; Clustalo Version 1.1.0, http://www. ebi.ac.uk/Tools/msa/clustalo/). The multiple sequence alignment result was subsequently submitted to the sequence-structure homology recognition (FUGUE) server (Shi et al. 2001; FUGUE version 2.0 Profile Library Search Against HOMSTRAD, http://tardis.nibio.go.jp/fugue/prfsearch.html). Results with ZSCORE $\geq 6$ have confidence levels $\geq 99 \%$.

\section{Antibody preparation, tissue distribution, and GST pull-down assay}

Custom polyclonal anti-CRNF antibodies were made against the epitope CSHRNANCQNDCFDIEFGKVKPR. For apCRNF tissue distribution, homogenates from Aplysia pleural-pedal ganglia, heart, body wall, and buccal mass were subjected to Western blot analysis using anti-apCRNF antibody (1:5000). GST pull-down assays were performed as described in Reissner et al. (2008).

\section{Cell culture, apCRNF treatment, and assay for neurite growth}

Cell culture was performed as described in Zhao et al. (2009) and Reissner et al. (2010). Sensory neurons from pleural ganglia and LFS motor neurons from abdominal ganglia were used. Control (vehicle alone) and apCRNF treatment were always performed on SNs from the same animal in parallel. Recombinant apCRNF $(250 \mathrm{ng} / \mathrm{mL})$ or vehicle $(0.1 \%$ BSA in PBS) was added on cultured SNs at Day 3 in vitro and incubated for $3 \mathrm{~d}$. Bright field images were captured with a Zeiss Axiovert 200 microscope. Twenty evenly spaced concentric circles with increasing radii from 30 to 315 $\mathrm{mm}$ were overlaid onto the center of a cell soma; neurite elonga- tion and branchings were measured by Sholl analysis (Sholl 1953; Gensel et al. 2010).

\section{Immunocytochemistry}

Immunocytochemistry of cultured SNs or MNs was performed as described by Lyles et al. (2006) using anti-apCRNF antibody (1:1000) and Alexa-633 goat anti-rabbit antibody (1:1000). Images were acquired with an Olympus Fluoview confocal laserscanning microscope.

\section{Semi-quantitative RT-PCR and fluorescence in situ hybridization (FISH)}

Pleural and pedal ganglia cDNA was prepared and semi-quantitative PCR was performed as described in Reissner et al. (2008). To ensure that amplification was kept within linear range, the number of cycles was optimized for each primer pair. apCRNF sense or antisense riboprobes were generated by in vitro transcription from SP6 or T7 promoters. FISH of cultured SNs or MNs was performed as described in Lyles et al. (2006). Images were acquired with an Olympus Fluoview laser-scanning microscope.

\section{apCRNF immunosorbent assay (ELISA)}

Isolated pleural-pedal ganglia were desheathed to expose the somata of SNs/MNs. For each animal, one pair of pleural-pedal ganglia was assigned to the experimental group and the other pair to control. Ganglia were incubated with $\mathrm{KCl}+5 \mathrm{HT}(\mathrm{KCl}$, $150 \mathrm{mM} ; 5 \mathrm{HT}, 50 \mu \mathrm{g} / \mathrm{mL}$ ) or ASW for $15 \mathrm{~min}$. Plates were coated with the conditioned medium samples, blocked with $0.5 \%$ BSA, and washed. Subsequently, the primary antibody (anti-apCRNF antibody, 1:5,000) was added and incubated overnight at $4^{\circ} \mathrm{C}$. After washing, the secondary antibody (alkaline phosphataseconjugated secondary anti-rabbit, 1:4,000) was added and incubated for $1.5 \mathrm{~h}$ at room temperature. The ELISA samples were then developed with para-nitrophenylphosphate and the absorbance was obtained with ThermoLabSystems Multiskan ascent spectrophotometer.

\section{MAPK activation}

Isolated pleural-pedal ganglia were desheathed to expose the somata of SNs/MNs. For each animal, one pair of pleural-pedal ganglia was assigned to the experimental group and the other pair to control. Ganglia were incubated with apCRNF (500 ng/ $\mathrm{mL}$ ) or vehicle (0.1\% BSA in PBS) for $2.5 \mathrm{~h}$, a single TNS (30 V, $40 \mathrm{~Hz}, 5-\mathrm{msec}$ shock duration, 1-sec train duration) was delivered to one pair of pleural-pedal ganglia in the experimental group, followed by another 1-h treatment of either apCRNF or ASW. For genistein experiments, bilateral pleural-pedal ganglia from the same animal were incubated with $100 \mu \mathrm{M}$ genistein (Purcell et al. 2003) or vehicle $(0.33 \%$ DMSO $/ 0.43 \%$ ethanol) for $30 \mathrm{~min}$, followed by application of apCRNF $(500 \mathrm{ng} / \mathrm{mL})$ for an additional $30 \mathrm{~min}$. A single TNS $(30 \mathrm{~V}, 40 \mathrm{~Hz}, 5$-msec shock duration, 1-sec train duration) was delivered to one pair of pleural-pedal ganglia, followed by another 1-h treatment of apCRNF and either genistein or vehicle. Immediately after the treatment, SN clusters were excised, lysed, and subjected to Western blot. Blots were probed with a total p44/42 MAPK antibody and a phospho p44/p42 MAPK antibody, and developed using chemiluminescence or Li-cor Odyssey imaging systems.

\section{Synaptic facilitation}

Intracellular recordings were made from $\mathrm{SN}-\mathrm{MN}$ coculture as described in Reissner et al. (2010). Baseline EPSPs were recorded before treatment. Coculture was then exposed to a $2.5-\mathrm{h}$ treatment of either vehicle or apCRNF $(500 \mathrm{ng} / \mathrm{mL})$, coterminated with a 5-min 5HT (10 mM) pulse, followed by another 1-h treatment of either vehicle or apCRNF $(500 \mathrm{ng} / \mathrm{mL})$, and incubated in culture medium overnight at $18^{\circ} \mathrm{C}$. LTF at the $\mathrm{SN}-\mathrm{MN}$ synapses was tested 18-24 h later. 


\section{Data analysis}

Unless indicated, all the data were presented as mean \pm SEM. All $t$-tests and post hoc tests used are two-tailed. Nonparametric statistics were used in Figures 5B and 6 due to the non-normality of the data. When $P<0.05$, the differences were considered significant.

\section{Statistical analysis of neurite growth}

For individual cells, a curve was generated by plotting crossings versus ring numbers (as in Fig. 2C). The area under the curve was then obtained by integration. The data in Figure 2D were summarized as the average areas of the control group versus apCRNF-treated group. Between-group comparisons were performed using unpaired $t$-tests.

\section{Statistical analysis of ELISA}

The data were normalized to the mean value of baseline measurement (ASW), and one-tailed paired $t$-test was performed for between-group comparisons (ASW vs. $\mathrm{KCl}+5 \mathrm{HT}$ ).

\section{Statistical analysis of MAPK activation}

The experimental ganglia phospho-MAPK versus total-MAPK ratio was normalized to the within-animal control ganglia phospho versus total MAPK ratio. Paired $t$-tests or a nonparametric Wilcoxin matched-pairs signed rank test (due to the nonnormality of the data for the genistein experiments [Fig. 5B]) was used for within-group comparisons between experimental ganglia and the corresponding control. A one-way ANOVA followed by Fischer's LSD procedure were used for between-group comparisons.

\section{Statistical analysis of physiology}

EPSP amplitudes were normalized to the baseline measurement. Data are presented as median \pm IQR. A Wilcoxon Signed Rank test was used for within-group comparisons due to non-normality of the data. Kruskal-Wallis test followed by Dunn's multiple comparison procedure were used for between-group comparisons.

\section{Acknowledgments}

We thank past and present members of the Carew Lab for very helpful comments on an earlier draft, Dr. Kathryn J. Reissner for help cloning apCRNF, and Dr. Kelsey Martin for use of an Aplysia CNS library. We also thank S. Fischbach, X. Ye, D. Anderson, M. Avakian, S. Khorasani, A. Lee, E. Patel, A. Pham, M. Rajani, R. Seese, and G. Shenasan for their help with the experiments. This work was supported by NIMH R01MH041083 and R01MH094792 and NSF Grant IOB-0444762 to T.J.C. We also thank the Optical Biology Core facility of the Developmental Biology Center at UC Irvine.

\section{References}

Atkins CM, Selcher JC, Petraitis JJ, Trzaskos JM, Sweatt JD. 1998. The MAPK cascade is required for mammalian associative learning. Nat Neurosci 1: $602-609$.

Bailey CH, Kandel ER. 2008. Synaptic remodeling, synaptic growth and the storage of long-term memory in Aplysia. Prog Brain Res 169: 179-198.

Bailey CH, Bartsch D, Kandel ER. 1996. Toward a molecular definition of long-term memory storage. Proc Natl Acad Sci 93: 13445-13452.

Beck G, Munno DW, Levy Z, Dissel HM, Van-Minnen J, Syed NI, Fainzilber M. 2004. Neurotrophic activities of trk receptors conserved over 600 million years of evolution. J Neurobiol 60: 12-20.

Bibel M, Hoppe E, Barde YA. 1999. Biochemical and functional interactions between the neurotrophin receptors trk and p75NTR. EMBO J 18: 616-622.

Carew TJ, Menzel R, Shatz CJ. 1998. Points of contact between development and learning. In Mechanistic relationships between development and learning (ed. Carew TJ, Menzel R, Shatz CJ), pp. 1-14. John Wiley \& Sons, Berlin.
Chao MV. 2003. Neurotrophins and their receptors: A convergence point for many signalling pathways. Nat Rev Neurosci 4: 299-309.

Chin J, Angers A, Cleary LJ, Eskin A, Byrne JH. 2002. Transforming growth factor $\beta 1$ alters synapsin distribution and modulates synaptic depression in Aplysia. J Neurosci 22: RC220.

Cohen-Cory S, Kidane AH, Shirkey NJ, Marshak S. 2010. Brain-derived neurotrophic factor and the development of structural neuronal connectivity. Dev Neurobiol 70: 271-288.

Fainzilber M, Smit AB, Syed NI, Wildering WC, Hermann PM, van der Schors RC, Jimenez C, Li KW, van Minnen J, Bulloch AG, et al. 1996. CRNF, a molluscan neurotrophic factor that interacts with the p75 neurotrophin receptor. Science 274: 1540-1543.

Gensel JC, Schonberg DL, Alexander JK, McTigue DM, Popovich PG. 2010. Semi-automated Sholl analysis for quantifying changes in growth and differentiation of neurons and glia. J Neurosci Methods 190: $71-79$.

Hu JY, Glickman L, Wu F, Schacher S. 2004. Serotonin regulates the secretion and autocrine action of a neuropeptide to activate MAPK required for long-term facilitation in Aplysia. Neuron 43: 373-385.

Huang EJ, Reichardt LF. 2003. Trk receptors: Roles in neuronal signal transduction. Annu Rev Biochem 72: 609-642.

Kassabov SR, Choi YB, Karl KA, Vishwasrao HD, Bailey CH, Kandel ER. 2013. A single aplysia neurotrophin mediates synaptic facilitation via differentially processed isoforms. Cell Rep 3: 1213-1227.

Kopec AM, Carew TJ. 2013. Growth factor signaling and memory formation: Temporal and spatial integration of a molecular network. Learn Mem 20: 531-539.

Levi-Montalcini R, Angeletti PU. 1968. Nerve growth factor. Physiol Rev 48: $534-569$.

Lyles V, Zhao Y, Martin KC. 2006. Synapse formation and mRNA localization in cultured Aplysia neurons. Neuron 49: 349-356.

Marinesco S, Carew TJ. 2002. Serotonin release evoked by tail nerve stimulation in the CNS of Aplysia: Characterization and relationship to heterosynaptic plasticity. J Neurosci 22: 2299-2312.

McIlroy G, Foldi I, Aurikko J, Wentzell JS, Lim MA, Fenton JC, Gay NJ, Hidalgo A. 2013. Toll-6 and Toll-7 function as neurotrophin receptors in the Drosophila melanogaster CNS. Nat Neurosci 16: 1248-1256.

McKay SE, Purcell AL, Carew TJ. 1999. Regulation of synaptic function by neurotrophic factors in vertebrates and invertebrates: Implications for development and learning. Learn Mem 6: 193-215.

Ormond J, Hislop J, Zhao Y, Webb N, Vaillaincourt F, Dyer JR, Ferraro G, Barker P, Martin KC, Sossin WS. 2004. ApTrkl, a Trk-like receptor, mediates serotonin-dependent ERK activation and long-term facilitation in Aplysia sensory neurons. Neuron 44: 715-728.

Philips GT, Tzvetkova EI, Carew TJ. 2007. Transient mitogen-activated protein kinase activation is confined to a narrow temporal window required for the induction of two-trial long-term memory in Aplysia. J Neurosci 27: 13701-13705.

Philips GT, Ye X, Kopec AM, Carew TJ. 2013. MAPK establishes a molecular context that defines effective training patterns for long-term memory formation. J Neurosci 33: 7565-7573.

Poo MM. 2001. Neurotrophins as synaptic modulators. Nat Rev Neurosci 2: $24-32$.

Purcell AL, Sharma SK, Bagnall MW, Sutton MA, Carew TJ. 2003. Activation of a tyrosine kinase-MAPK cascade enhances the induction of long-term synaptic facilitation and long-term memory in Aplysia. Neuron 37: 473-484.

Reissner KJ, Shobe JL, Carew TJ. 2006. Molecular nodes in memory processing: Insights from Aplysia. Cell Mol Life Sci 63: 963-974.

Reissner KJ, Boyle HD, Ye X, Carew TJ. 2008. Aplysia synapse associated protein (APSAP): Identification, characterization, and selective interactions with Shaker-type potassium channels. J Neurochem 105: 1006-1018.

Reissner KJ, Pu L, Schaffhausen JH, Boyle HD, Smith IF, Parker I, Carew TJ. 2010. A novel postsynaptic mechanism for heterosynaptic sharing of short-term plasticity. J Neurosci 30: 8797-8806.

Sharma SK, Sherff CM, Shobe J, Bagnall MW, Sutton MA, Carew TJ. 2003. Differential role of mitogen-activated protein kinase in three distinct phases of memory for sensitization in Aplysia. J Neurosci 23: 3899-3907.

Sharma SK, Sherff CM, Stough S, Hsuan V, Carew TJ. 2006. A tropomyosin-related kinase B ligand is required for ERK activation, long-term synaptic facilitation, and long-term memory in Aplysia. Proc Natl Acad Sci 103: 14206-14210.

Sherff CM, Carew TJ. 2004. Parallel somatic and synaptic processing in the induction of intermediate-term and long-term synaptic facilitation in Aplysia. Proc Natl Acad Sci 101: 7463-7468.

Shi J, Blundell TL, Mizuguchi K. 2001. FUGUE: Sequence-structure homology recognition using environment-specific substitution tables and structure-dependent gap penalties. J Mol Biol 310: 243-257.

Shobe JL, Zhao Y, Stough S, Ye X, Hsuan V, Martin KC, Carew TJ. 2009. Temporal phases of activity-dependent plasticity and memory are 
mediated by compartmentalized routing of MAPK signaling in Aplysia sensory neurons. Neuron 61: 113-125.

Sholl DA. 1953. Dendritic organization in the neurons of the visual and motor cortices of the cat. J Anat 87: 387-406.

Sievers F, Wilm A, Dineen D, Gibson TJ, Karplus K, Li W, Lopez R, McWilliam H, Remmert M, Soding J, et al. 2011. Fast, scalable generation of high-quality protein multiple sequence alignments using Clustal Omega. Mol Syst Biol 7: 539.

Sutton MA, Ide J, Masters SE, Carew TJ. 2002. Interaction between amount and pattern of training in the induction of intermediate- and long-term memory for sensitization in Aplysia. Learn Mem 9: 29-40.

Vicario-Abejon C, Owens D, McKay R, Segal M. 2002. Role of neurotrophins in central synapse formation and stabilization. Nat Rev Neurosci 3: 965-974.

Wiesmann C, de Vos AM. 2001. Nerve growth factor: Structure and function. Cell Mol Life Sci 58: 748-759.
Ye X, Shobe JL, Sharma SK, Marina A, Carew TJ. 2008. Small G proteins exhibit pattern sensitivity in MAPK activation during the induction of memory and synaptic facilitation in Aplysia. Proc Natl Acad Sci 105: 20511-20516.

Zhang F, Endo S, Cleary LJ, Eskin A, Byrne JH. 1997. Role of transforming growth factor- $\beta$ in long-term synaptic facilitation in Aplysia. Science 275: $1318-1320$.

Zhao Y, Wang DO, Martin KC. 2009. Preparation of Aplysia sensory-motor neuronal cell cultures. J Vis Exp 28: e1355.

Zhu B, Pennack JA, McQuilton P, Forero MG, Mizuguchi K, Sutcliffe B, Gu CJ, Fenton JC, Hidalgo A. 2008. Drosophila neurotrophins reveal a common mechanism for nervous system formation. PLoS Biol 6: e284.

Received October 21, 2013; accepted in revised form February 5, 2014. 


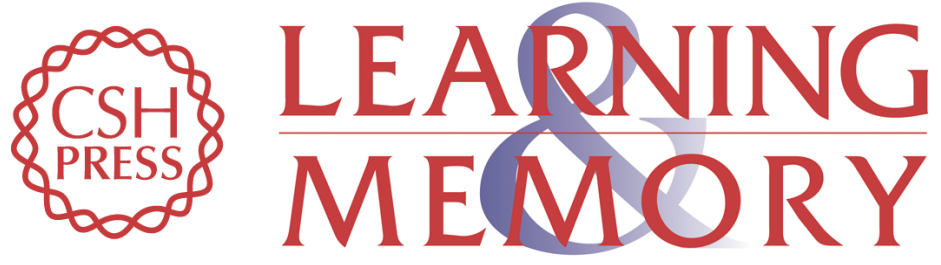

\section{A novel cysteine-rich neurotrophic factor in Aplysia facilitates growth, MAPK activation, and long-term synaptic facilitation}

Lu Pu, Ashley M. Kopec, Heather D. Boyle, et al.

Learn. Mem. 2014, 21:

Access the most recent version at doi:10.1101//m.033662.113

\section{Supplemental http://learnmem.cshlp.org/content/suppl/2014/03/07/21.4.215.DC1 Material}

References This article cites 41 articles, 16 of which can be accessed free at: http://learnmem.cshlp.org/content/21/4/215.full.html\#ref-list-1

Creative This article is distributed exclusively by Cold Spring Harbor Laboratory Press for the Commons first 12 months after the full-issue publication date (see

License http://learnmem.cshlp.org/site/misc/terms.xhtml). After 12 months, it is available under a Creative Commons License (Attribution-NonCommercial 4.0 International), as described at http://creativecommons.org/licenses/by-nc/4.0/.

Email Alerting Receive free email alerts when new articles cite this article - sign up in the box at the Service top right corner of the article or click here. 